\title{
O papel do enfermeiro preceptor na residência de enfermagem em uma instituição
}

\section{militar}

The role of the precepting nurse in the nursing residence in a military institution

El papel de la enfermera preceptante en la residencia de enfermería de una institución militar

Recebido: 09/04/2021 | Revisado: 16/04/2021 | Aceito: 17/04/2021 | Publicado: 02/05/2021

Tuane Carla Moreira da Silva

ORCID: https://orcid.org/0000-0002-0966-4036 Universidade Federal do Estado do Rio de Janeiro, Brasil

E-mail: silva.enftesm@yahoo.com.br

Pâmela Oliveira de Andrade

ORCID: https://orcid.org/0000-0003-3821-6838 Universidade Federal do Estado do Rio de Janeiro, Brasil

E-mail: pamela.oliveira.andraade@ hotmail.com

Andréia Jorge da Costa

ORCID: https://orcid.org/0000-0001-6923-4401 Universidade Federal do Estado do Rio de Janeiro, Brasil

E-mail: andreiajcosta@msn.com

Sônia Regina de Souza

ORCID: https://orcid.org/0000-0001-7981-0038 Universidade Federal do Estado do Rio de Janeiro, Brasil

E-mail: sonia.souza@unirio.br

Vinícius Rodrigues de Souza

ORCID: https://orcid.org/0000-0001-8035-3647

Universidade Federal Fluminense, Brasil

E-mail: viniciussouza.enf@gmail.com

Geilsa Soraia Cavalcanti Valente

ORCID: https://orcid.org/0000-0003-4488-4912

Universidade Federal Fluminense, Brasil

E-mail: geilsavalente@gmail.com

Inez Silva de Almeida

ORCID: https://orcid.org/0000-0001-5082-5607

Universidade Estadual do Rio de Janeiro, Brasil

E-mail: inezalmeida2016@gmail.com

\begin{abstract}
Resumo
Objetivo: Compreender o papel do enfermeiro preceptor no processo de treinamento em serviço dos residentes de enfermagem. Metodologia: Estudo qualitativo, descritivo e exploratório, em que participaram onze enfermeiras de um hospital militar. Utilizou-se para coleta de dados um questionário semiestruturado contendo perguntas relacionadas as atribuições e percepções dos preceptores junto aos residentes de enfermagem. Os dados foram analisados e agrupados em quatro categorias temáticas, sendo discutidos à luz de estudiosos ligados à temática. Resultados: Compreendeu-se que o preceptor é um mediador do conhecimento e formação profissional de residentes, adota estratégias de ensinoaprendizagem para facilitar a troca de conhecimentos e estabelece relações pautadas no respeito mútuo, na confiança e na autonomia de seus alunos. Conclusão: A instituição de saúde precisa incentivar o aprimoramento de competências e habilidades dos preceptores a fim de alcançar a excelência do processo de ensino, uma vez que o conhecimento não é estático e a educação deve acompanhar as inovações científicas e exigências do mercado de trabalho e sociedade.
\end{abstract}

Palavras-chave: Preceptoria; Enfermagem; Educação em saúde; Papel do profissional de enfermagem; Ensino.

\begin{abstract}
Objective: To understand the role of the preceptor nurse in the in-service training process of nursing residents. Methodology: Qualitative, descriptive and exploratory study, in which eleven nurses from a military hospital participated. A semi-structured questionnaire containing questions related to the preceptors' attributions and perceptions with nursing residents was used for data collection. The data were analyzed and grouped into four thematic categories, being discussed in the light of scholars linked to the theme. Results: It was understood that the preceptor is a mediator of knowledge and professional training of residents, adopts teaching-learning strategies to facilitate the exchange of knowledge and establishes relationships based on mutual respect, trust and autonomy of his students. Conclusion: The health institution needs to encourage the improvement of competences and skills of
\end{abstract}


preceptors in order to achieve excellence in the teaching process, since knowledge is not static and education must accompany scientific innovations and the demands of the labor market and society.

Keywords: Preceptorship; Nursing; Health education; Nurse's role; Teaching.

\section{Resumen}

Objetivo: Comprender el papel de la enfermera preceptora en el proceso de formación en servicio de los residentes de enfermería. Metodología: Estudio cualitativo, descriptivo y exploratorio, en el que participaron once enfermeras de un hospital militar. Para la recolección de datos se utilizó un cuestionario semiestructurado que contiene preguntas relacionadas con las atribuciones y percepciones de los preceptores con los residentes de enfermería. Los datos fueron analizados y agrupados en cuatro categorías temáticas, siendo discutidos a la luz de estudiosos vinculados al tema. Resultados: Se entendió que el preceptor es un mediador del conocimiento y la formación profesional de los residentes, adopta estrategias de enseñanza-aprendizaje para facilitar el intercambio de conocimientos y establece relaciones basadas en el respeto mutuo, la confianza y la autonomía de sus alumnos. Conclusión: La institución de salud necesita incentivar la mejora de las competencias y habilidades de los preceptores para lograr la excelencia en el proceso de enseñanza, ya que el conocimiento no es estático y la educación debe acompañar las innovaciones científicas y las demandas del mercado laboral y la sociedad.

Palabras clave: Preceptoría; Enfermería; Educación en salud; Rol de la enfermera; Ensenãnza.

\section{Introdução}

No ano de 1940 deu-se início aos primeiros Programas de Residência, como modalidade de ensino e aprendizagem em serviço, sendo criada em 1960 a Residência de Enfermagem, regulamentada pela Resolução do Conselho Federal de Enfermagem. (Silva, 2017) Esse modelo de curso, consiste em uma modalidade de treinamento em serviço, possibilitando ao estudante aprimorar a prática assistencial remunerada em serviço, através da vivência diária nos cenários de saúde, associada ao conhecimento teórico adquirido ao logo do curso de graduação. Deste modo, segundo Avila et al., (2017) o enfermeiro residente, na maioria das vezes, recém-formado, adquire experiência e aprimoramento de habilidades técnicas e saberes no campo de prática.

Nas Unidades de Treinamento em Serviço (UTS) são construídos os cenários de aprendizagem prática, os quais mobilizam os elementos indispensáveis para os saberes teóricos e habilidades pertinentes à profissão, que consideram os quatro pilares da educação, de acordo com a Lei das Diretrizes e Bases da Educação Nacional - LDB 9394/96 (Brasil, 1996): aprender a (re) conhecer, aprender a fazer, aprender a se relacionar e aprender a ser. Nesse contexto de formação em serviço, o preceptor é o responsável por desempenhar o papel fundamental de conectar a teoria à prática (Santos \& Noro, 2017), realizando a supervisão direta das atividades práticas desenvolvidas pelos residentes nos serviços de saúde.

Segundo o Ministério da Educação (2012), a preceptoria é exercida pelo profissional de nível superior de saúde, que tenha domínio dos instrumentos práticos e teóricos de sua profissão. Para melhor compreensão, através do disposto na Resolução da Comissão Nacional de Residência Multiprofissional em Saúde - CNRMS refere-se à função do preceptor:

“Art.13 A função de preceptor caracteriza-se por supervisão direta das atividades práticas realizadas pelos residentes nos serviços de saúde onde se desenvolve o programa, exercida por profissional vinculado à instituição formadora ou executora, com formação mínima de especialista”.

Além disso, Ferreira et al., (2018) enfatizam que o preceptor também deve se preocupar em auxiliar e identificar as fragilidades de aprendizado, estimular a participação do residente e do graduando, propiciar a aplicação de conhecimento teórico nas atividades práticas, participar no planejamento e execução de atividades, estimular a autoaprendizagem. Por fim, caso necessário, deve buscar apoio junto aos demais docentes e tutores, com o intuito de sanar as possíveis fragilidades apresentadas por estes estudantes. (Autonomo et al., 2015) 
Para Fonseca et al., (2020), com uma nova dimensão dos serviços nos processos de aprendizagem e novas formas de ensinar e aprender surge a necessidade de um preceptor atualizado, e que viva o cotidiano dos serviços, acompanhando o desenvolvimento do aprendizado, problematizando e favorecendo a produção do conhecimento de forma inovadora, crítica e reflexiva.

Sendo assim, a amplitude e a importância do tema envolvendo a preceptoria, apresenta-se diretamente relacionada à vivência do enfermeiro no âmbito de suas atribuições, contribuindo dessa forma para o ensino, pesquisa e assistência de Enfermagem. Deste modo, este estudo visa compreender o papel do enfermeiro preceptor durante o processo de treinamento em serviço aos residentes de enfermagem.

\section{Metodologia}

Trata-se de um estudo qualitativo, descritivo e exploratório, orientado pela seguinte questão norteadora: "Qual é o papel do enfermeiro preceptor no Curso de Residência em Enfermagem?". Para Ludke e Andre (2013), a pesquisa qualitativa se desenvolve em uma situação natural, rico em dados descritivos, com um plano aberto e flexível e focalizado na realidade de forma complexa e contextualizada. O cenário da pesquisa foi um hospital escola de natureza militar, localizado no Estado do Rio de Janeiro, que se constitui como campo de prática para o Curso de Residência em Enfermagem da Universidade Federal do Estado do Rio de Janeiro (UNIRIO). Este estudo é oriundo de um Trabalho de Conclusão de Residência em Enfermagem da UNIRIO, tendo sido aprovado pelo Comitê de Ética e pesquisa do Hospital Naval Marcílio Dias sob o parecer n 3.969.541.

Como instrumento de coleta de dados, foi aplicado um questionário semiestruturado com 11 preceptoras de enfermagem. As participantes foram orientadas em relação a origem das perguntas, bem como os objetivos das mesmas. $\mathrm{O}$ questionário foi estruturado em duas partes. Na primeira, foram perguntadas informações como sexo, idade, tempo de atuação, formação complementar com a finalidade de melhor caracterizar os participantes do estudo. Na segunda, foram realizadas as seguintes perguntas: 1) Em qual momento assumiu função de preceptoria? 2) Participou de alguma capacitação para atuação de preceptores? 3) Quais estratégias de ensino aprendizagem realiza com os residentes de enfermagem? 4) Como pensa ser a relação pessoal entre preceptor e aluno?

Para garantir o anonimato, foi atribuído um código numérico às participantes, denominado pela letra "E" de enfermeiro, e por algarismos arábicos de acordo com a ordem de realização das entrevistas. Logo, as participantes foram identificadas como E1, E2, E3, sucessivamente.

Para a interpretação e análise dos dados, optou-se pela análise temática por ser a mais apropriada para pesquisas em saúde, desdobrando-se nas etapas de pré-análise, exploração do material ou codificação e tratamento dos resultados obtidos/ interpretação. (Minayo, 1996)

Analisando as respostas dos sujeitos, foram destacados núcleos de sentidos relacionados ao objeto estudado, derivando em 03 categorias temáticas: Inserção do enfermeiro na função de preceptoria; As estratégias de ensino-aprendizagem implantadas pelos preceptores durante o treinamento em serviço; e o Acolhimento e a relação estabelecida entre o enfermeiro preceptor e o residente de enfermagem. Os achados foram estudados, discutidos e analisados à luz de estudiosos ligados à temática.

\section{Resultados e Discussão}

Todas as entrevistadas foram do sexo feminino, com idade média de 40 anos (mínimo de 29 anos e máximo de 56 anos). As participantes possuíam atuação profissional entre um período de 6 a 29 anos, tendo especializações em áreas diversas, tanto assistenciais quanto gerenciais. 
Em relação a formação complementar, apenas uma participante possuía Pós-Graduação Stricto Sensu em nível de Mestrado. As demais enfermeiras relataram possuir Pós-Graduação Lato Sensu em diversos segmentos da Enfermagem. Cabe ressaltar que não foi identificada nenhuma preceptora com especialização inserida na temática de ensino e docência. Diante do exposto e das 03 categorias temáticas desenvolvidas, é possível observar a fala dos preceptores envolvidos na formação de enfermeiros residentes, conforme apresentados a seguir:

\section{Categoria I: Inserção do enfermeiro na função de preceptoria}

Essa categoria objetivou demonstrar o momento de ingresso das participantes na função de preceptoria. Foi possível observar que nove enfermeiras iniciaram a função de preceptoria através de determinações da chefia, não tendo sido ofertado a possibilidade de escolha para tal atribuição. Rego Filho e Santos (2018) apontam a necessidade de formação para qualificar os preceptores e sugere ser do serviço essa responsabilidade.

Outrossim, cabe destacar que o profissional atuante no Sistema Único de Saúde (SUS) possui responsabilidades de contribuir e atuar nas atividades de saúde, não só de assistência ao paciente, mas também como preceptor de alunos de diversos níveis de formação.

Nesse contexto, Ribeiro et al., (2020) reforçam sobre a colaboração da Política Nacional de Educação Permanente que propõe que os processos de capacitação do pessoal da saúde sejam estruturados a partir da problematização do seu processo de trabalho e que tenham como objetivo a transformação das práticas profissionais e da própria organização do trabalho.

No âmbito da capacitação realizada para atuação de preceptores, apenas uma preceptora relatou ter recebido treinamento específico na área de docência.

Fui inserida no momento que fui nomeada a encarregada do serviço. (E2.)

Devido a assunção de cargo de encarregada da enfermaria. (E4.)

Foi uma determinação do Departamento de Enfermagem. (E7.)

Obtive um curso preparatório para a inserção no campo prático dos residentes. (E9.)

Compreende-se que o enfermeiro desempenha importantes tarefas na sua prática profissional, sendo primordial manter uma estreita interação entre universidade e serviço de saúde. Esse profissional acumula atividades voltadas à assistência, gestão de pessoas e materiais, atividades educativas, entre outras. Logo, fragilidades no processo de ensinoaprendizagem poderão ocorrer, principalmente quando esse profissional não possui uma capacitação prévia para a preceptoria. Neste sentido, destacam-se importantes falas:

O tempo destinado a parte de educação permanente está diminuindo no setor devido ao quadro reduzido de enfermeiros. (E1.)

São muitas atividades a realizar, e as vezes não consigo oferecer a atenção merecida para ensinar (E10).

Existe a necessidade de uma capacitação para atuarmos como preceptoras, pois trabalhar no âmbito do ensino exige um saber peculiar. (E11.) 
Embora o papel do preceptor seja conhecido, muitas vezes esta função torna-se desafiadora em seu cotidiano. Com isso, o profissional poderá reproduzir o ensino de acordo com as vivencias prévias no campo de trabalho (Antunes, 2016), sem refletir sobre uma necessidade de aprimoramento metodológico do ensino.

Nesse contexto, Souza e Ferreira (2019) reforçam que o preceptor deverá contemplar conhecimentos que vão além de uma formação generalista, humanista, crítica e reflexiva. Será necessário qualificar-se constantemente, a fim de incentivar o enfermeiro residente a desenvolver responsabilidades e compromisso social, permeados por rigor técnico, científico e ético. Deste modo, os alunos desenvolverão um olhar humanizado e compatível com o cenário em que serão coadjuvantes neste processo de ensino-aprendizagem e qualificação profissional.

O preceptor ao executar um procedimento com a intenção de demonstrá-lo para o aluno deverá empenhar-se de forma qualificada. Sendo assim, acredita-se que esse promotor da educação de novos profissionais, necessita conhecer e desenvolver metodologias educacionais para articular e estimular o saber técnico e científico destes alunos, tendo em vista que os residentes são profissionais em formação.

Nesse contexto, cabe salientar que, ainda que alguns enfermeiros tenham cursado a licenciatura na área, não se pode considerar que se tornem especialistas em preceptoria, pois essa tarefa exige o domínio de elementos intervenientes na capacidade de ensinar, como competências, conhecimentos e habilidades. Em concordância, Ferreira et al., (2018) enfatizam que os preceptores precisam aprender a ter atitude de estarem sempre refletindo quanto aos seus saberes e competências profissionais, num processo interno de autoaprendizagem.

Sobre a aquisição de saberes, habilidades e competências, algumas participantes corroboram que:

O enfermeiro preceptor deve possuir competência técnica, conhecimento científico e didático e atualização constante. (E1.)

Além de ser um profissional com experiência e competência na área de atuação, o enfermeiro preceptor deve ser um profissional com perfil para receber residentes e ter disponibilidade para ensinar. (E10.)

No que tange a aquisição de competências, Perrenoud (2000) define que é a faculdade de mobilizar um conjunto de recursos cognitivos (saberes, capacidades, informações, etc.) para solucionar com pertinência e eficácia uma série de situações. Sendo assim, para o cumprimento da missão de preceptoria, torna-se necessário uma capacitação prévia, de modo que o enfermeiro reconheça as demandas dessa função, diante da importância de ser um sujeito propagador do conhecimento e desenvolvedor de habilidades. Logo, depreende-se que a competência pedagógica é essencial para qualificar o desempenho do preceptor em sua prática, bem como a aplicabilidade de meios e estratégias de ensino-aprendizagem, para otimizar o processo educativo de discentes.

\section{Categoria II: As estratégias de ensino-aprendizagem implementadas pelos enfermeiros preceptores durante o treinamento em serviço}

Historicamente, o ensino na saúde tem sido pautado no uso de métodos tradicionais, colocando o professor no centro do processo educativo, como transmissor de conteúdo, e o aluno um mero expectador, passivo e repetidor (SILVA et al., 2018). Entretanto, muito tem se discutido sobre uma ampla transformação na educação de profissionais de saúde, objetivando uma melhor qualidade do ensino e um egresso com visão crítica e reflexiva.

$\mathrm{Na}$ enfermagem, já se evidencia o uso de diversas tecnologias incorporadas às metodologias de ensino existentes, empregadas pelos docentes, tanto por meio de equipamentos e softwares utilizados em laboratórios, quanto por técnicas de 
ensino diferenciadas. Entretanto, sendo ferramentas complementares ao ensino, e não como único método utilizado (Pissaia et al., 2017).

Com essa nova dimensão dos serviços nos processos de aprendizagem, Magalhães et al., (2020) afirmam que surge a necessidade de um profissional vinculado e que viva o cotidiano dos serviços, acompanhando o desenvolvimento do aprendizado, problematizando os saberes e favorecendo a produção do conhecimento. Assim, a função do preceptor é essencial para a formação de outro tipo de profissional, que atenda as demandas de um novo mercado de trabalho.

Para além do âmbito da formação, e considerando que o conhecimento é um processo de construção coletiva, o exercício da preceptoria deve promover a troca de saberes entre preceptores, residentes e usuários. Logo, para efetivar o que foi posto, Antunes (2016) explica que é uma exigência necessária que a comunicação entre profissionais, preceptores e residentes seja eficaz, ampliando a resolubilidade das ações em saúde e educação e evitando a possibilidade de omissões e duplicações de cuidado. Nessa perspectiva, a habilidade de comunicação e o trabalho interprofissional de diversas áreas surgem como uma oportunidade real para a integração e troca de saberes, para a construção do fazer coletivo.

Como exemplo prático da necessidade de novas formas de ensinar, observa-se nas falas das participantes do estudo, os momentos e as estratégias utilizadas para a promoção do conhecimento e reflexão sobre a prática:

Através da discussão dos casos passados durante a passagem de plantão, visitas e mediante dúvidas dos residentes. (E7.)

A associação acontece no decorrer da prática do residente através da observação direta (procedimento técnico executado, evoluções, diagnósticos e condutas tomadas pelo residente) e discussões/indagações/apresentações acerca de algum tema específico. (E9.)

Corroborando com o exposto, observa-se que nesse momento de aprendizagem, também há a formação de conceitos, de valores científicos e humanitários dos alunos. Com isso, Antunes (2016) reitera que os serviços de saúde, exigem do enfermeiro preceptor uma atenção redobrada, presteza e resolubilidade em ações e tomada de decisões, bem como condutas éticas, tão presentes na profissão do cuidar. Sob esse prisma, uma das depoentes sinaliza que:

Tento utilizar diversas ferramentas para repassar o conhecimento que possuo, mas sempre tomo muito cuidado com a exposição do paciente e com as questões éticas. (E8.)

Nesse exercício de preceptoria, estimula-se também a reflexão dos residentes em suas práticas e melhorias contínuas nos espaços de trabalho. Em contrapartida, as atividades de ensino possibilitam reflexões que favorecem o crescimento profissional nos cenários de formação. Logo, consideraram-se 03 importantes falas, que exemplificam outras estratégias de ensino e de aprendizagem utilizadas:

Questionamentos à beira do leito, discussão dos casos dos pacientes internados no setor, solicitando a busca das respostas quando o residente não sabe. (E3.)

É importante que os residentes tragam assuntos para relacionar a teoria e prática. A prática só pode ser realizada conhecendo a teoria. Sempre procuro orientar as ações relacionadas com a teoria. (E5.) 
A associação acontece no decorrer da prática do residente, através da observação direta (procedimento técnico executado, evoluções, diagnósticos e condutas tomadas pelo residente) e discussões/indagações/apresentações acerca de algum tema específico. (E7.)

Com isso, Avila et al., (2017) e Freitas et al., (2015) relatam que é importante reconhecer que a formação profissional está fundamentada em estratégias de ensino-aprendizagem que devem ser implementadas nos programas de residência em parceria com os campos de prática para fortalecer a qualificação profissional do aluno e do preceptor, voltadas para o trabalho em conjunto, e para o compartilhamento de ideias do trabalho em equipe, potencializando o intercâmbio de saberes e práticas.

\section{Categoria III: Acolhimento e a relação estabelecida entre o enfermeiro preceptor e o residente de enfermagem}

De acordo com o Ministério da Saúde (2011), acolhimento é definido como o ato ou o efeito de acolher, uma ação de aproximação, um "estar com" e um "estar perto de", ou seja, uma atitude de inclusão que implica estar em relação com algo ou alguém. Logo, é de suma importância que o preceptor acolha o residente realizando uma escuta ativa de seus anseios e aflições diante do novo momento de sua formação. Dessa forma, os alunos se sentem parte do processo do cuidado e adquirem segurança nas execuções de suas tarefas diárias.

Para Seltenreich (2017), estar disponível para ensinar é mostrar-se receptivo para também aprender, pois esses são processos que se complementam e não podem ser vistos dissociados. Assim, pode-se considerar, que quem ensina também aprende, tratando-se de uma relação que se estabelece e produz aprendizagens.

Sobre a relação entre o preceptor e o residente de enfermagem, as participantes destacaram aspectos indispensáveis nessa interação entre agentes do conhecimento:

Deve ser estabelecida uma relação de troca lensino e aprendizagem. (E5.)

Deve ser uma relação construtiva entre dois profissionais de saúde. (E6.)

Uma boa relação havendo confiança mútua entre as partes. (E10.)

Compreende-se a necessidade de relação de vínculo entre os elementos envolvidos no ato de ensinar, em prol do bemestar comum e do trabalho em equipe. O sucesso de uma relação entre preceptores e residentes perpassa um processo de comunicação eficaz, pautada no respeito, no compromisso, na disponibilidade, na confiança mútua e nas atitudes que devem orientar as propostas de educação em saúde.

Sabe-se que desafios estão presentes na formação de alunos, de forma interligada: o desenvolvimento pessoal, a expectativa profissional, os cenários de aprendizagem diversificados, as tarefas complexas, a busca por desempenho resolutivo e o sucesso profissional. Permeando os itens citados, ocorrem relações entre o preceptor e seu residente, as quais tudo se conecta.

Entendo a importância de um bom acolhimento e relação pessoal com os residentes, pois os mesmos chegam com muitos medos, expectativas e planejamentos. (E4.)

Nesse processo de construção de identidade profissional do enfermeiro residente, a relação estabelecida com o preceptor precisa ser pedagógica, por ser desenvolvida em momentos de ensinar e aprender. Silva (2017) infere que o contato 
interpessoal que se estabelece entre ambos, as estratégias de ensino empregadas pelo preceptor e as vivências nos cenários de aprendizagem, contribuirão para a qualificação de profissionais com formação crítica e reflexiva, voltados para as exigências de um perfil profissional atuante no mercado de trabalho.

Assim, o Curso da Residência de Enfermagem favorece ao aluno enfermeiro a vivência de experiências variadas, o autoconhecimento, o reconhecimento das falhas e sucessos, bem como a ampliação de domínio do conhecimento técnico e científico. Como consequência, atinge-se à formação de um profissional com uma visão crítica e gerencial da assistência a ser prestada, com tomada de decisões visando o atendimento de excelência e segurança do paciente.

\section{Conclusão}

Este estudo permitiu a compreensão da importância dos meios de ensino nos cenários de aprendizagem, das relações estabelecidas entre o enfermeiro preceptor e o enfermeiro residente e do conhecimento para o sucesso do processo de ensinoaprendizagem no Curso da Residência em Enfermagem. Além disso, demonstrou que a figura do preceptor representa a luz de um farol no processo educativo de alunos, que estes seguirão como referência em suas jornadas profissionais.

O enfermeiro preceptor possui uma importante atuação como mediador do conhecimento de enfermeiros residentes. O profissional precisa ter conhecimentos técnicos e científicos e habilidades para ensinar, de forma a possibilitar meios de ensino e o estímulo necessário aos educandos, no cenário de aprendizagem. Considerando que o conhecimento não é estático, torna-se necessário uma atualização constante para compreender os processos de inovações científicas e tecnológicas, bem como as estratégias de ensino aprendizagem, para o seu desempenho profissional e ensino, junto aos enfermeiros residentes.

Portanto, novos estudos e produções científicas tornam-se necessários para que o conhecimento seja sempre ressignificado, a fim de proporcionar reflexões e adoção de novas ações, com vistas às inovações das práticas educativas. Logo, pode-se afirmar que a excelência do trabalho educativo de preceptores durante a qualificação de enfermeiros, com vistas às inovações científicas e tecnológicas, proporciona a formação de novos profissionais com competências para atuar, frente às necessidades de saúde exigidas pela sociedade e pelo mercado de trabalho.

Em suma, sugere-se que esses novos estudos tragam um aprimoramento à formação qualificada dos preceptores, garantindo dessa forma um egresso com reflexões críticas e reflexivas diante da sua prática profissional. Além do exposto, é necessário pensar em pesquisas que trabalhem a aplicabilidades de novas metodologias de ensino na relação entre preceptores e residentes, visto que essa dinâmica é constituída em dupla via, no qual ambas as partes são contempladas com a construção de um novo saber.

\section{Referências}

Antunes, J. D. M. (2016). A preceptoria na formação do residente em Enfermagem em Saúde Coletiva: o aprender e o ensinar no cotidiano do Sistema Único de Saúde. Dissertação de mestrado, Universidade Federal Fluminense. https://pesquisa.bvsalud.org/portal/resource/pt/biblio-836773

Autonomo, F. R. D. O. M., Hortale, V. A., Santos, G. B. D., \& Botti, S. H. D. O. (2015). A Preceptoria na formação médica e multiprofissional com ênfase na atenção primária-Análise das Publicações Brasileiras. Revista Brasileira de Educação Médica,39(2), 316-327. https://doi.org/10.1590/1981$52712015 \mathrm{v} 39 \mathrm{n} 2 \mathrm{e} 02602014$

Avila, R. S. S., Penna, M. A., Pinno, C., Durgante, V. L., Saul, A. M. R., Farão, E. M. D., \& de Lima, S. B. S. (2017). Vivên cias de residentes enfermeiros no programa de residência multiprofissional em saúde. Saúde (Santa Maria), 43(1), 13-21. https://doi.org/10.5902/2236583414826

Brasil (1996). Ministério da Educação. Lei 9394 de 20 de dezembro de 1996. Estabelece as diretrizes e bases da educação nacional. http://www.planalto.gov.br/ccivil_03/leis/19394.htm

Brasil (2012). Comissão Nacional de Residência Multiprofissional em Saúde. Resolução CNRM No 2 de 13 de abril de 2012 . Dispõe sobre as Diretrizes Gerais para os Programas de Residência Multiprofissional e em Profissional de Saúde. http://portal.mec.gov.br/residencias-em-saude/residencia-multiprofissional

Brasil (2011). Ministério da Saúde. Secretaria de Atenção à Saúde. Departamento de Atenção Básica. Acolhimento à demanda espontânea. https://aps.saude.gov.br/biblioteca/visualizar/MTIwNQ== 
Farias, B. C. D. S. S., \& Noro, L. R. A. (2017). PET-Saúde como indutor da formação profissional para o Sistema Único de Saúde. Ciência \& Saúde Coletiva, 22, 997-1004. http://dx.doi.org/10.1590/1413-81232017223.15822016

Ferreira, F. D. C., Dantas, F. D. C., \& Valente, G. S. C. (2018). Nurses' knowledge and competencies for preceptorship in the basic health unit. Revista brasileira de enfermagem, 71,1564-1571. http://dx.doi.org/10.1590/0034-7167-2016-0533.

Fonseca, J. P., Macedo, E., Machado, C. C., \& Caregnato, R. C. A. (2020). Training of precepts in the Multiprofissional or Uniprofissional residence in the health area: an integrative review. Research, Society and Development, 9(8), e699986299. https://doi.org/10.33448/rsd-v9i8.6299

Freitas, C. M., Freitas, C. A. S. L., Parente, J. R. F., Vasconcelos, M. I. O., Lima, G., Mesquita, K. O., Martins, S. C. \& Mendes, J. D. R. (2015). Uso de metodologias ativas de aprendizagem para a educação na saúde: análise da produção científica. Trabalho, Educação e Saúde, 13(2), 117-130.

Ludke, M. \& Andre, M. E. D. A. (2013). Pesquisas em educação: uma abordagem qualitativa. E.P.U.

Magalhães, M.S.C., Souza, A.C. \& Azevedo, GM. (2020). Contributions of Nursing Preceptorship in the context of Primary Health Care from the perspective of active methodologies. Research, Society and Development, 9(7): 1-18, e270973681. http://dx.doi.org/10.33448/rsd-v9i7.3681

Minayo, M. C. D. S. (1996). O desafio do conhecimento: pesquisa qualitativa em saúde. In O desafio do conhecimento: pesquisa qualitativa em saúde (pp. 407-p).

Perrenoud, P. (2000). Dez novas competências para ensinar; trad. Patrícia Chittoni Ramos. Artmed.

Pissaia, L. F., da Costa, A. E. K., Rehfeldt, M. J. H., \& Moreschi, C. (2017). Tecnologia educacional no processo de formação de enfermeiros. Cinergis, 18(3), 185-189. http://dx.doi.org/10.17058/cinergis.v18i3.8865

Rego Filho, J. F., \& Santos, C. S. (2018). Identifying the Profiles and Activities of Preceptors in a Nursing Residency Program TT - Residência Em Enfermagem: Identificação Das Atividades de Preceptoria Em Um Hospital Escola. Mundo Saúde, 42(2), 333-48. https://doi.org/10.15343/0104-780920184202333348

Ribeiro, P. K. C., Firmo, W. da C. A., Sousa, M. H. S. L., Figueiredo, I. A., \& Pacheco, M. A. B. (2020). Os profissionais de saúde e a prática de preceptoria na atenção básica: assistência, formação e transformações possíveis. JMPHC | Journal of Management \& Primary Health Care. 1-18. https://doi.org/10.14295/jmphc.v12.977

Santos, B. C. S. F., \& Noro, L. R. A. (2017). PET-Saúde como indutor da formação profissional para o Sistema Único de Saúde. Ciência e Saúde Coletiva, 22(3), 997-1004. http://dx.doi.org/10.1590/1413-81232017223.15822016

Seltenreich, L. D. S. (2017). Competências do enfermeiro na prática da preceptoria na residência multiprofissional em saúde. Dissertação de mestrado, Universidade Estadual Paulista Júlio de Mesquita Filho.https://repositorio.unesp.br/handle/11449/150278

Silva, R. M., Silva Freitas, L., Araújo, C. L. S., Camargo, J., Franco, A. M., Silva, J. N., \& Ferreira, I. P. (2018). Importância da residência em enfermagem no processo ensino-aprendizagem: uma revisão integrativa. Revista Enfermagem Atual In Derme, 86(24). https://doi.org/10.31011/reaid-2018-v.86-n.24-art.120

Silva, V. C. (2017). Os saberes que emergem da prática social do enfermeiro preceptor na residência multiprofissional em saúde. Tese de Doutorado, Universidade Federal do Rio de Janeiro.. http://objdig.ufrj.br/51/teses/850375.pdf

Souza, S. V., \& Ferreira, B. J. (2019). Preceptoria: perspectivas e desafios na Residência Multiprofissional em Saúde. ABCS Health Sciences, 44(1). https://doi.org/10.7322/abcshs.v44i1.1074 